\title{
COMPARISON OF TURBULENT KINETIC ENERGY IN THE WAKE REGION OF MODIFIED CIRCULAR CYLINDERS
}

\author{
HAMIDREZA SHIRI \& HASAN HACISEVKI \\ Department of Mechanical Engineering, Eastern Mediterranean University, Turkey
}

\begin{abstract}
Flow properties and wake structures behind bluff bodies was one of the interesting topics between scholars for many years. Such interest in this phenomenon is due to various applications of bluff bodies in engineering and industry. In this study a comparison of flow structures of the wake of circular cylinder, semi-circular and $\mathrm{c}$ shape cylinders have been presented. The wake flows have been compared in terms of shedding frequency, Reynolds stress and turbulent kinetic energy (TKE) to identify any similarities between these geometries. It was found that the highest value of TKE occurred on either side of the separated shear layer. Moreover, it was observed that as the TKE develops in the downstream the peaks were shifted towards centerline in the wake region.

Keywords: vortex shedding, turbulent kinetic energy, circular cylinder, semi-circular cylinder, Strouhal number.
\end{abstract}

\section{INTRODUCTION}

Formation of vortex street in the wake region of bluff bodies is a matter of great importance in the field of engineering. Such periodic phenomenon, incur destructive loading on the structural body and may lead to catastrophic incidents. Therefore, to prevent such destruction, it requires a better understanding of this phenomenon. As a result, numerous scientists dedicated their research on this phenomenon to investigate bluff bodies such as circular cylinder, square cylinder or flat plate [1]-[4]. In this context, circular cylinders is one of the most investigated geometries due to its vast possible industrial applications. These researches investigate circular cylinder at various arrangements such as single, tandem, or staggered with various Reynolds number. While the purpose of these studies are focused on a better understanding of vortex shedding phenomenon behind circular cylinder and possible methods to suppress the vortex street in the wake region, finding any possible similarities of similar geometries is an interesting topic. For instance, Ozgoren [5] investigated flow structure in the wake of circular and square cylinder at incident angle of 45 degree. The Strouhal numbers observed to be in range $\mathrm{St}=0.204-0.212$ for the circular cylinder, $\mathrm{St}=0.12-0.134$ for the square cylinder and $\mathrm{St}=0.165-0.174$ for the square cylinder at incidence. The vortex shedding frequency in the wake region of elliptical and square cylinders studied by Knauss et al. [6]. It was reported that the while Strouhal number depended on the geometrical shape of the bluff body, but almost independent of Reynolds number. In addition, Ozgoren et al. [7] and Provansal et al. [8] investigated flow characteristics in the wake region behind circular cylinder and sphere. The study demonstrated that the wake flow of the sphere was more complex than the wake flow of the circular cylinder because of the 3-D flow. Furthermore, sphere exhibit higher vortex shedding frequency, while circular cylinder demonstrates higher power spectral density (PSD). It was also reported that turbulence kinetic energy peak in the wake of circular cylinder is three times higher than peak values in wake of sphere. Hacışevki and Teimourian [9] compared the wake region behind two identical tandem plates and square cylinders. They observed a lower Strouhal number in wake region behind square cylinder than the wake region of tandem 
plates. This research intended to study possible similarities of the wake region features. To identify flow similarities the wake region have been studied in terms of vortex shedding frequency, coherent structures and a quantitative comparison for variation of turbulent kinetic energy (TKE) have been presented.

\section{EXPERIMENTAL SETUP}

Investigations conducted in an open-return subsonic wind tunnel at Mechanical Engineering Department, Eastern Mediterranean University, Turkey. The free stream velocity of the experiment was $U_{\infty}=10.44 \pm 2 \% \mathrm{~m} / \mathrm{s}$. The Reynolds number of the experiment was $\operatorname{Re}=20,660\left(R e=\frac{U_{\infty} D}{v}\right)$ based on cylinder diameter of $30 \mathrm{~mm}$. In this study flow structures around a semi-circular cylinders (SC) and a circular cylinder (CC) with a diameter of $30 \mathrm{~mm}$ were investigated. In addition, a $\mathrm{C}$ shape cylinder (CS) with a diameter of $30 \mathrm{~mm}$ was also considered to identify flow structure similarities between these geometries. Stream wise and transverse velocity components have been acquired by employing TSI Flow point 1,500 CTA Hot-Wire anemometer system for various $\mathrm{x} / \mathrm{D}$ ratios behind the plates. The domain of investigation in the wake region was between $1.0 \mathrm{D}$ to $6.0 \mathrm{D}$ of the cylinder diameter $(1.0 \leq \mathrm{x} / \mathrm{D} \leq 6.0)$ as illustrated in Fig. 1.

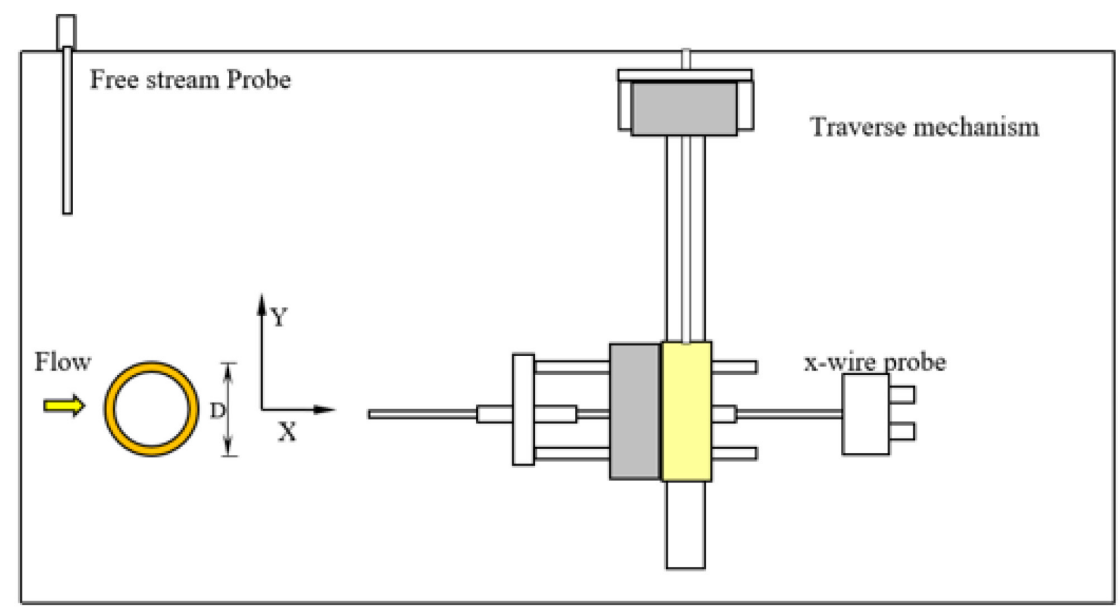

Figure 1: Experimental setup and coordinates: top view.

The flow properties have been analyzed by triple decomposition method and ensemble averaging techniques. Therefore, any instantaneous properties of flow can be defined as superposition of time-mean averaged component $\bar{u}$, coherent component $\tilde{u}$ and incoherent component as follows

$$
\varphi=\bar{\varphi}+\tilde{\varphi}+\varphi^{\prime} .
$$

For phase averaging technique, the operation will be performed on successive data having the same phase with respect to a specific wave. Therefore

$$
\left\langle\varphi^{\prime}\right\rangle=\frac{1}{n} \sum_{1}^{n} \varphi_{i}^{\prime},
$$


where $n$ is the number of cycles used for phase averaging and $\left\langle\phi^{\prime}\right\rangle$ is the value of incoherent component at the same normalized time successive cycles. The triple decomposition method and phase averaging techniques implemented on the Navier-Stokes momentum equation and as a result a modified Navier-Stokes equation obtained.

\section{RESULTS AND DISCUSSION}

The dominant vortex shedding frequency can be determined by implementing Fast Fourier Transformation (FFT) on the acquired velocity component in the domain of investigation. Therefore, the spectral peak will indicate the dominant vortex shedding frequency. Spectral analysis of transverse velocity components in the wake of modified circular cylinders is illustrated in Fig. 2.

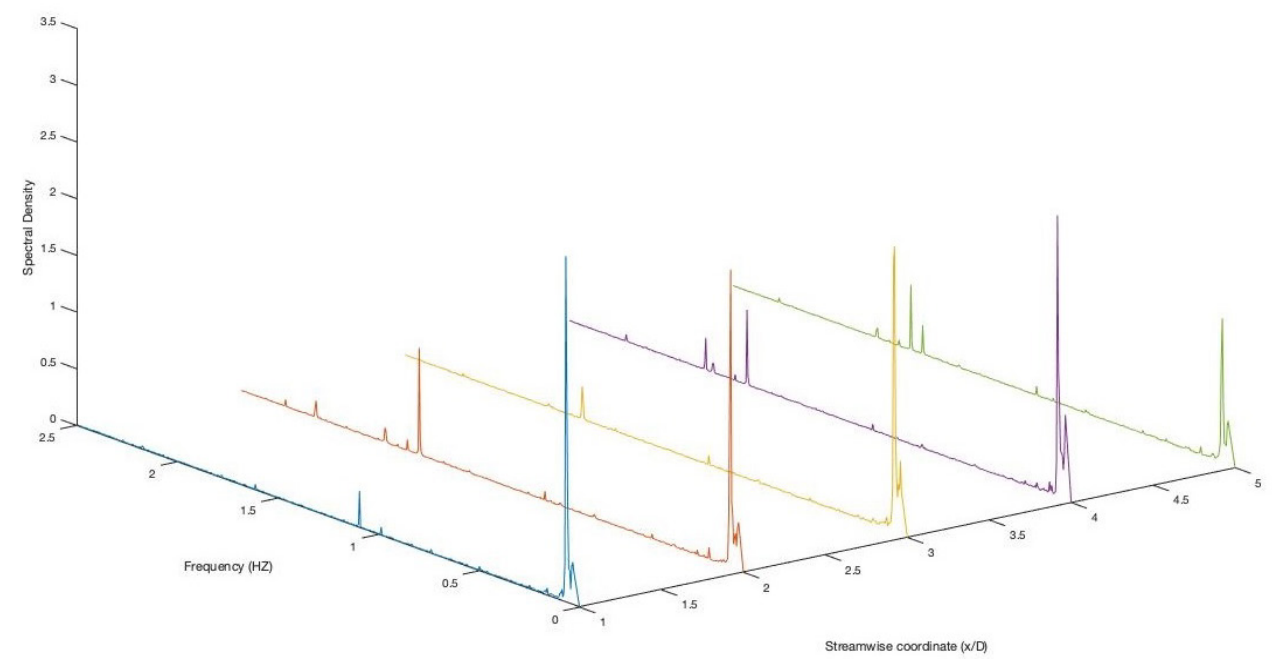

Figure 2: Spectral analysis for various $\mathrm{x} / \mathrm{D}$ behind semi-circular cylinder.

From Fig. 2 the dominant shedding frequency is apparent as a single peak. Which correspond to an identical Strouhal number $\left(s_{t}=f D / U_{\infty}\right)$ for all $\mathrm{x} / \mathrm{D}$ 's near wake behind the cylinders and have shown in Table 1 . The FFT analysis on both stream wise and transverse velocity components resulted in equal dominant shedding frequency and for sake of brevity only transverse results were presented.

Table 1: Frequency and Strouhal number for different geometries.

\begin{tabular}{|l|c|c|}
\hline Geometry & Frequency $(\mathrm{Hz})$ & $\mathrm{S}_{\mathrm{t}}$ \\
\hline Circular cylinder $(\mathrm{CC})$ & 63.5 & 0.182 \\
\hline Semi-circular cylinder $(\mathrm{SC})$ & 68.4 & 0.197 \\
\hline C shape cylinder $(\mathrm{CS})$ & 68.4 & 0.197 \\
\hline
\end{tabular}

In this study coherent and incoherent turbulent energy production behind modified circular cylinders have been investigated and some remarkable features of this periodic flow have been studied. Fig. 3 illustrated the development of the coherent TKE in the downstream wake of CC, SC and CS cylinders. As it can be seen from Fig. 3 the evolution of the TKE is 
clearly evident for higher $\mathrm{x} / \mathrm{D}$ downstream the wake. Similarly for all investigated geometries, near the base the TKE peak concentrated at either sides of the separated shear layer corresponding the shed vortices.

However, the TKE peaks shifted toward the center of the wake as the probe moves further downstream. In this regards, SC demonstrated a delay in transition of the peaks from sides
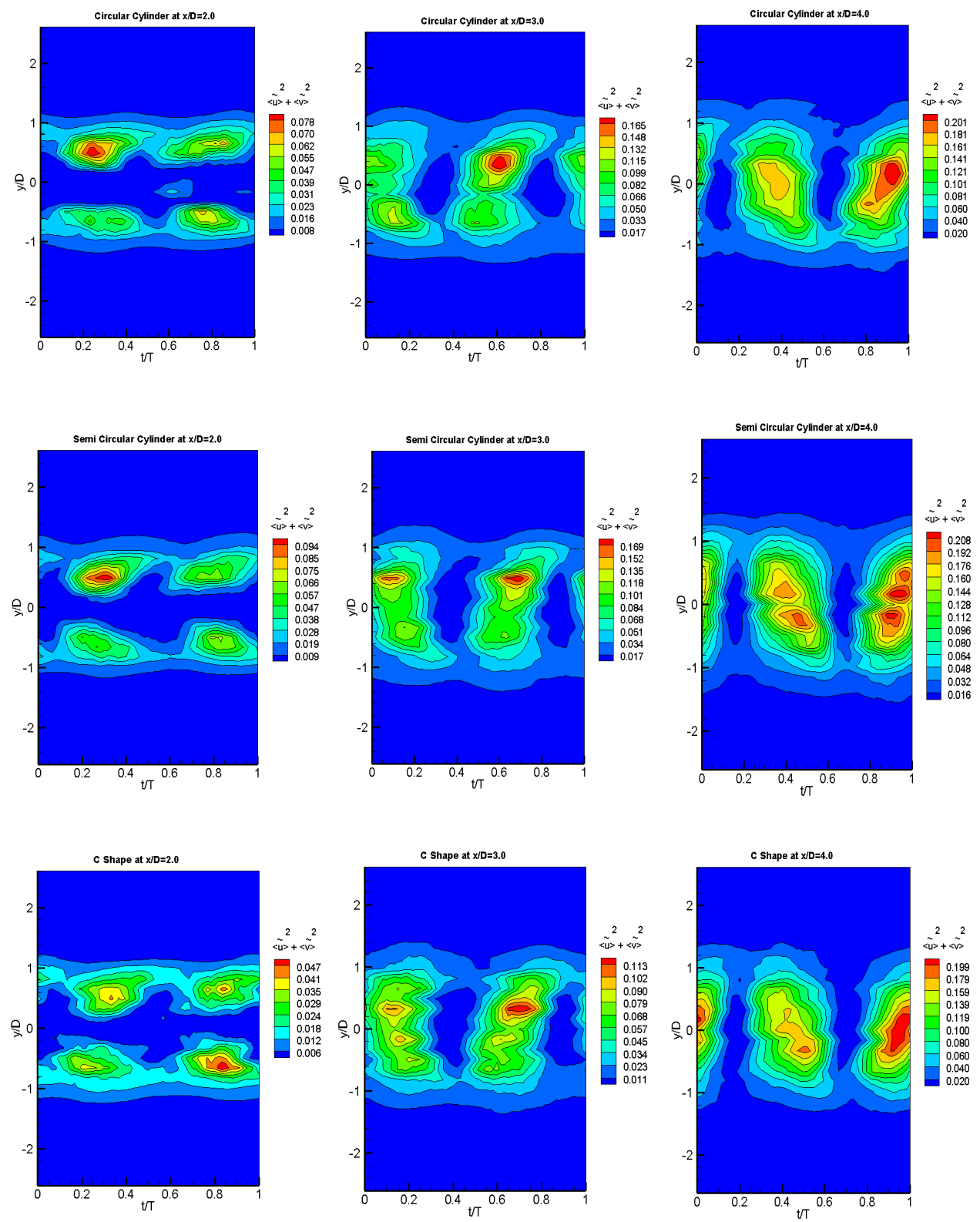

Figure 3: Coherent turbulent kinetic energy. 
of the wake towards the center. Therefore, in the region close to the base i.e. $x / D=2$ the TKE reaches its peak once per cycle at each sides of the wake, while at higher $\mathrm{x} / \mathrm{D}$ TKE peak occurs twice per cycle close the centerline of the wake. Fig. 4 demonstrated the incoherent turbulent energy for various $\mathrm{x} / \mathrm{D}$ in the wake region behind the modified circular cylinders. In a similar manner to coherent TKE as discussed previously, incoherent TKE development
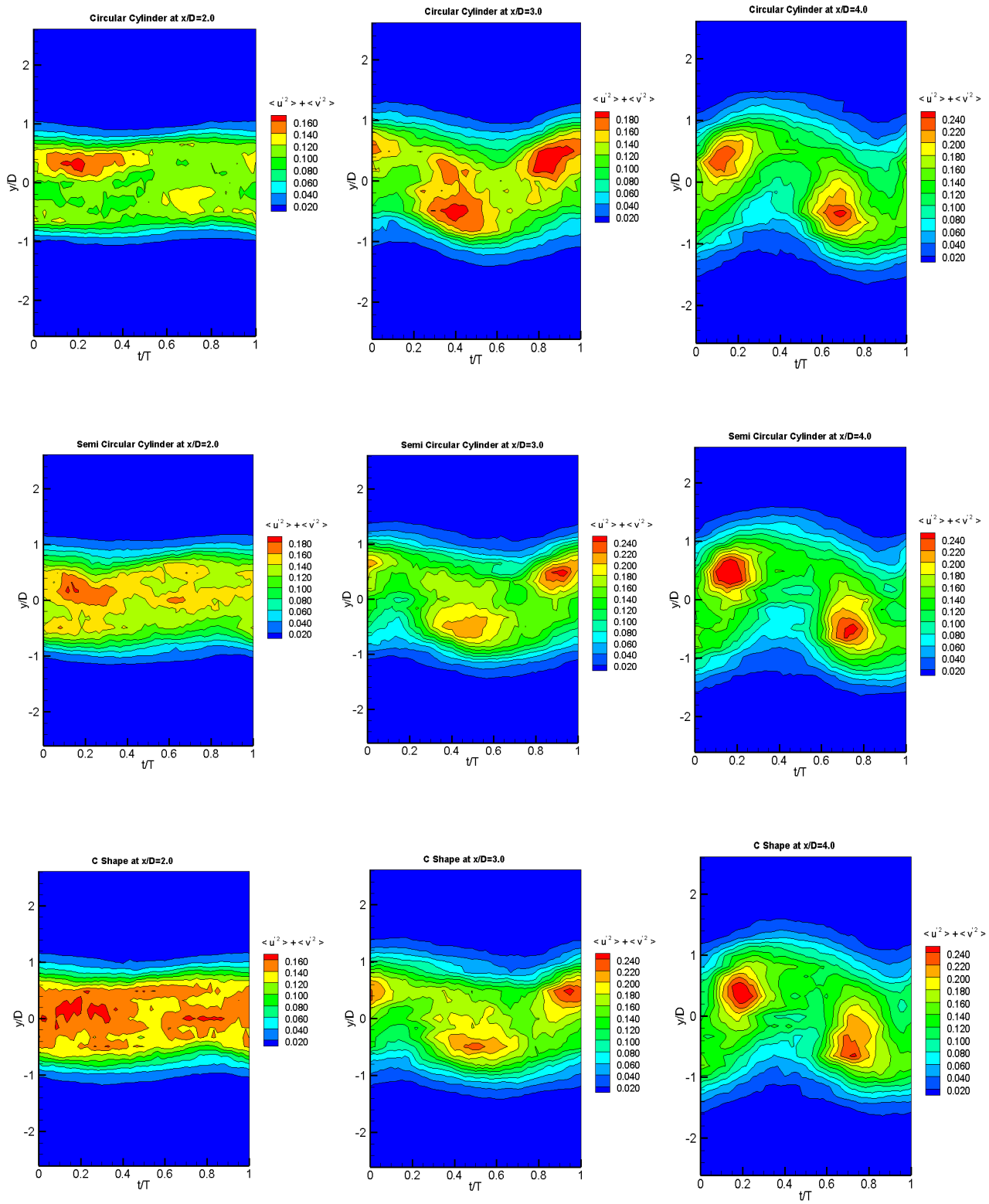

Figure 4: Incoherent TKE measured at different $\mathrm{x} / \mathrm{D}$ behind the cylinders. 
in the downstream wake is also evident from Fig. 4. Although, it has been demonstrated that, the incoherent TKE developed within the edges of the periodic flow as it pass towards downstream (Fig. 4). It was also observed that close to the base of the cylinder flow of periodic features were not apparent. However as the probe moves further the downstream in the wake region, the periodic features of flow developed and become more apparent.

Fig. 5 illustrated the time averaged of incoherent stream wise and transverse normal Reynolds stresses for various measured at $\mathrm{x} / \mathrm{D}=4.0$ in the wake region. From the figure a two peaks profile approximately corresponding to the edges of the cylinders are apparent for transverse stress. On the other hand, the stream wise stress reaches a peak alongside the wake center. Moreover, from the figure it can be concluded that the wake region behind the CC exhibited the lowest normal stresses compare to SC and CS.
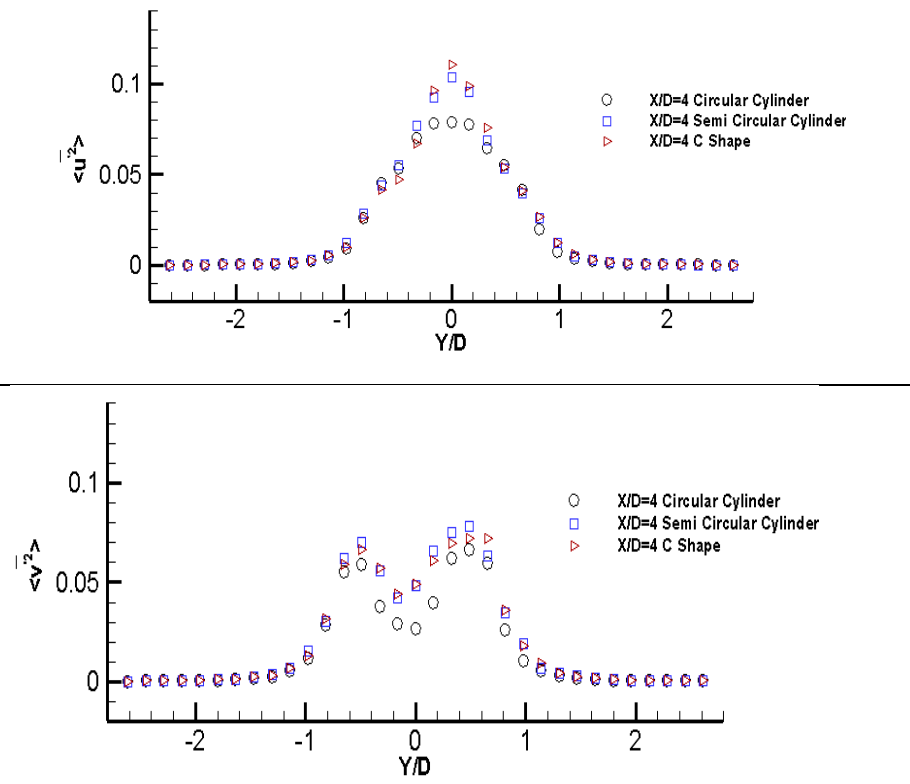

Figure 5: Time averaged incoherent normal Reynolds stress.

\section{CONCLUSION}

The flow structure in terms of Strouhal number and coherent and incoherent TKE in the downstream wake of modified circular cylinders have been investigated experimentally. It has been observed that the vortex shedding frequency behind semi-circular and $\mathrm{C}$ shaped have been increased by $7 \%$ respect to circular cylinder. From the plotted figures the evolution of the TKE production in the downstream wake was clearly evident. TKE for circular, semicircular, and $\mathrm{C}$ shape have been found from coherent max plot as $0.0858,0.1036$, and 0.0529 respectively. Therefore, it has been observed that the coherent TKE reduced by $38.3 \%$ in $\mathrm{C}$ shape cylinder with respect to circular cylinder near wake. On the other hand, TKE in the wake region for lower $\mathrm{x} / \mathrm{D}$ in semi-circle case has been increased by $20.7 \%$ with respect to circular cylinder. TKE is observed to be concentrated at sides of the separated shear layer for low $\mathrm{x} / \mathrm{D}$ 's whereas TKE peaks spotted at centerline in the wake region for higher $\mathrm{x} / \mathrm{D}$ 's in 
coherent case. Finally a double peaks profile corresponding to the edges of the cylinders have been observed for time averaged incoherent normal Reynolds stresses in transverse direction.

\section{REFERENCES}

[1] Chashechkin, Y.D. \& Zagumennyi, I.V., Formation of waves, vortices and ligaments in 2D stratified flows around obstacles. Physica Scripta, 94(5), 054003, 2019.

[2] Naumov, I. et al., Experimental investigation of wake evolution behind a couple of flat discs in a hydrochannel. Thermophysics and Aeromechanics, 23(5), pp. 657-666, 2016.

[3] Shin, B. \& Kondo, M., Effect of gap ratio on the wake behind two side-by-side flat plates. Journal of Applied Fluid Mechanics, 12(4), 2019.

[4] Teimourian, A., Hacisevki, H. \& Bahrami, A., Experimental study on flow past two inclined flat plates in tandem arrangement. Journal of Wind Engineering and Industrial Aerodynamics, 169, pp. 1-11, 2017.

[5] Ozgoren, M., Flow structure in the downstream of square and circular cylinders. Flow Measurement and Instrumentation, 17(4), pp. 225-235, 2006.

[6] Knauss, D.T., John, J. \& Marks, C., The vortex frequencies of bluff cylinders at low Reynolds numbers. Journal of Hydronautics, 10(4), pp. 121-126, 1976.

[7] Ozgoren, M. et al., Comparison of flow structures in the downstream region of a cylinder and sphere. International Journal of Heat and Fluid Flow, 32(6), pp. 1138-1146, 2011.

[8] Provansal, M., Schouveiler, L. \& Leweke, T., From the double vortex street behind a cylinder to the wake of a sphere. European Journal of Mechanics-B/Fluids, 23(1), pp. 65-80, 2004.

[9] Hacışevki, H. \& Teimourian, A., Comparison of flow structures in the wake region of two similar normal flat plates in tandem and a square cylinder. Experimental Thermal and Fluid Science, 69, pp. 169-177, 2015. 